\title{
A HOLISTIC APPROACH TO KNOWLEDGE MANAGEMENT AND SOCIAL LEARNING: LESSONS LEARNT FROM MILITARY HEADQUARTERS
}

\author{
Leoni Wame', Irena $\mathrm{Ali}^{\prime}$, Celina Pascoe ${ }^{2}$ and Katerina Agostino? \\ 'Defence Systems Analysis Division \\ Defence Science and Technology Organisation \\ Department of Defence \\ Canberra ACT 2600 \\ Lconi. Wameraidsio defoncc.gov.au \\ lrena.Ali(a) disto defence.gov.au \\ 2 Division of Communication and Education \\ University of Canberra ACT 260I \\ Celina.Pascoe@icomedu.camberra.edu.apu \\ 'Land Operations Division \\ Defence Science and Technology Organisation \\ Department of Defence \\ Edinburgh SA 5111 \\ katerina.agestino@dsio.defence.gov.au
}

\begin{abstract}
This paper reports on research conducted by the Enterprise Social Leaming Architecture (ESLA) team of the Defence Science and Technology Organisation. The ESLA team is investigating collaborative social leaming within the Australian Defence Organisation (ADO). Social leaming is tightly coupled to knowledge management. Three studies in three different settings have been conducted to date. The studies have provided multi-layered findings about social leaming, and validated the use of ethnography for this purpose. Preliminary findings are discussed in this paper in terms of identifiod enablers and motivators for effective social leaming and knowledge management. Although the paper deals with the defence environment. the findings can be generalised to other organisational settings, as the study deals with understanding the issues inherent in building sustainable and adaptive learning organisations.
\end{abstract}

\section{INTRODUCTION}

This paper reports the preliminary findings of research conducted by the Enterprise Social Learning Architecture (ESLA) team of the Defence Science and Technology Organisation. The ESLA task is currently in the third year of a four-year research study investigating social learning within the Australian Defence Organisation (ADO). A focus of the study has been to determine the factors that facilitate social learning and knowledge management within military command and control settings and, in so doing, to ascertain the elements of an environment that foster a seamless union between working and learning. To date, studies have been conducted in three different settings: a single service tactical headquarters, a joint strategic headquarters (all three services and civilians), and a singie service strategic headquarters (where the service is different to the service in the first study).

Social learning is defined as learning occurring within a group, an organisation, or any cultural cluster and includes: the procedures by which knowledge and practice are transmitted across posting cycles, across different work situations and across time; and the procedures that facilitate generative learning - learning that enhances the enterprise's ability to adjust to dynamic and unexpected situations and to react creatively to them.

The immediate aim of this research is to understand the issues inherent in building learning, adaptive and sustainable organisations. In this way, social learning is tightly coupled to knowledge management. The ESLA research team interprets knowledge management to be about the acquisition, construction, development, transfer, and sharing of the knowledge assets within an organisation in order to achieve the organisation's objectives. Knowledge management strategies develop organisational knowledge assets (people), support them by information technology and other tools, and enhance the processes by which individuals, groups, and the entire organisation learns. Although there are many tools and technologies, which may become an integral part of an organisation's knowledge management strategy, knowiedge management is primarily concerned with cultural, organisational, procedural,and human factors (Ali, 2000; Warne, 2000).

The ESLA research looks specifically at the cultural, organizational, procedural and human factors as applied to social learning.Findings have been used to derive a preliminary architecture that organisations might use to facilitate effective social learning for their staff. Results to date have identified a number of processes and strategies that enable and facilitate knowledge management and a selection of these factors is discussed in this paper. The long-term 
objective of this research, however, is to develop architectures that will support the development of information systems that guide and enhance organisational learning.

\section{METHODOLOGY}

Qualitative research techniques, in particular ethnographic approaches, have been used predominantly for this research because they are appropriate where a key aspect of the research is to analyse, or at least take into consideration, various aspects of the context and the social process (Harvey \& Myers, 1995). Ethnographers 'immerse' themselves into a situation for sufficient time to gradually see and understand the key factors that influence the setting being studied. It is most useful when researchers are attempting to understand complex functioning systems from a holistic perspective (Jordan, 1993) as it deals with real world practices in real world situations. Ethnography is often used, for example, to provide information systems researchers with rich insights into the human, social and organizational aspects of information system development and implementation (Harvey \& Myers, 1995). Given the exploratory nature of the research, as well as the importance of context in understanding the social process of learning, ethnographic techniques were considered to be the most suitable methodological tools for the study (Ali, 2000; Warne, 2000).

The primary form of ethnography used was 'field work', which entailed observing the work taking place in different settings, and using directed questioning to clarify issues. Currently the team comprises four researchers, with a wide range of backgrounds and specialisations, thereby enriching the collection and analysis of the data, and providing reliability for the findings. Regular team meetings ensure that findings are corroborated, consolidated and that emerging social learning issues are identified. To ensure validity, the collected data has been triangulated by source (different times and places), and, as the research progressed, by method (observations, interviews, surveys).

The methodologies used have evolved as the research has progressed from one study setting to another. While the methodology for the first setting was largely ethnographic, in subsequent settings, ethnographic observations were supplemented with quantitative surveys, and semi-structured interviews with a stratified sample of personnel in the area. All interviews were recorded and transcribed. The transcripts were then coded according to the thesaurus of terms specially developed by the ESLA team for coding of field observations and transcripts. The individual team members embedded these code terms, or textual labels, within the text of field notes, collected documents and interview transcripts. These embedded terms help the researcher to develop and map an analytical structure onto the data. Coding also allows the raw field data to be gathered together into more meaningful chunks so that they can be retrieved and analysed together (Miles \& Huberman, 1994). The overall aim is to discover the underlying patterns and regularities in the settings being studied.

\section{FINDINGS TO DATE}

The ESLA team has identified a number of environmental or cultural factors, processes and strategies that, when positively applied, facilitate social learning and knowledge management within the ADO. In this context, the environmental and cultural factors can be termed "motivators" and the processes and strategies termed "enablers". The motivators and enablers work together to generate an organisational environment that actively sustains and cultivates effective social learning. This environment both nurtures, and is nurtured by, a number of overriding organisational values that foster effective social leaming. There is a great deal of overlap and interlock among these factors, such that some enablers also motivate, some motivators enable and the organisational values both enable and motivate. The research team continues to seek better ways of representing these relationships, as evidenced in the paper on "Making the invisible visible" (Linger \& Warne, 2001), also in this issue. The research team has concluded that all of these broadly ranging enablers and motivators should ideally be operating together, in order to optimize social learning and knowledge management. Collectively, these factors give an organisation more capability and power than the sum of each of their component parts.

The research team also found that some of the same motivating and enabling factors could also act as inhibitors or challengers of social learning when they are not thoughtfully applied. In this way, while positive and appropriate application of motivators, enablers and values work together to form a holistic and effective social learning environment, the absence or inappropriate application of some of these factors can have a destabilizing and destructive impact on the effectiveness of organisational learning and knowledge assets. This is illustrated in the diagram below. 


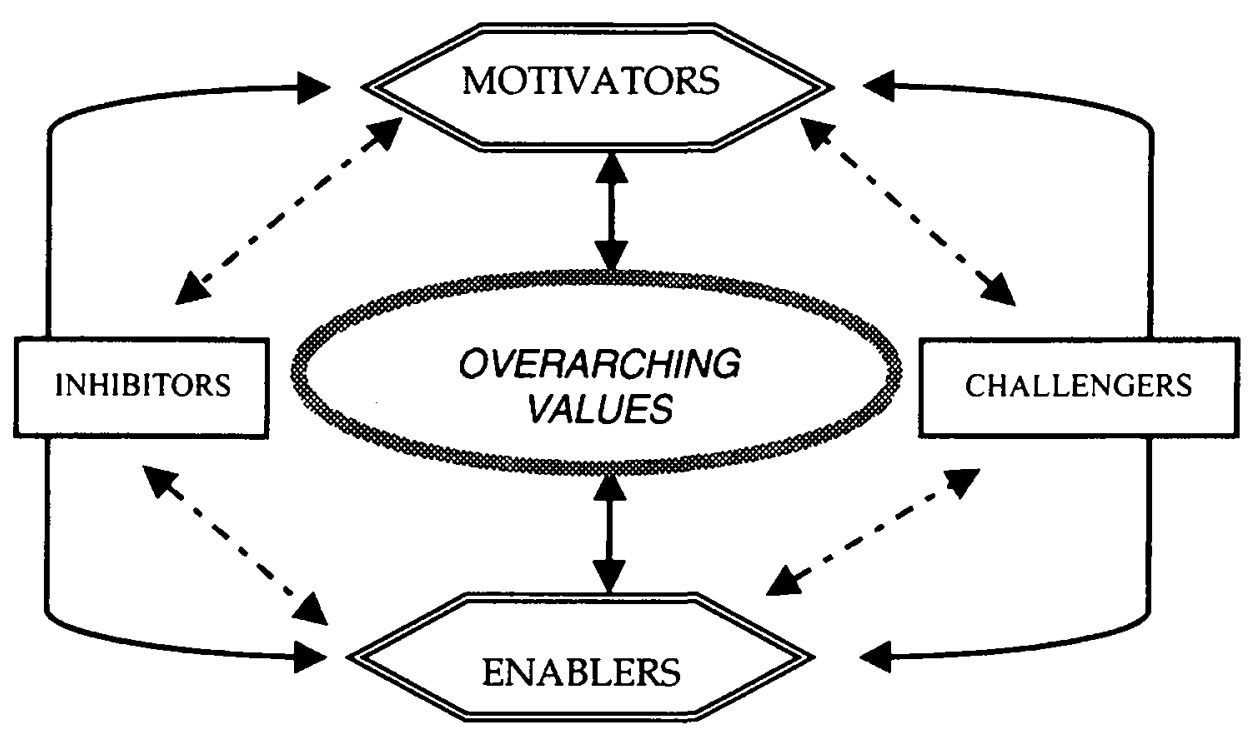

Figure 1 Factors In Social Learning

\section{ENABLERS AND MOTIVATORS}

The enablers and motivators discussed in this paper are representative of those identified from the data gathered so far and are by no means exhaustive. For ease of discussion, the identified enablers and motivators have been assigned to seven categories: Common Identity, Problem Solving, Team Building, Access to Information, Development of Individual Expertise, Communication, and, finally, Induction and Enculturation. Based on the ESLA research, these categories, or elements, are believed to be essential constructs for effective social learning (Ali, 2000; Warne, 2000). Further, the order in which they appear does not imply level of importance or value to social learning; however, each enabler was observed to have an impact on effectiveness of learning processes occurring in the settings under study (Figure 2). 


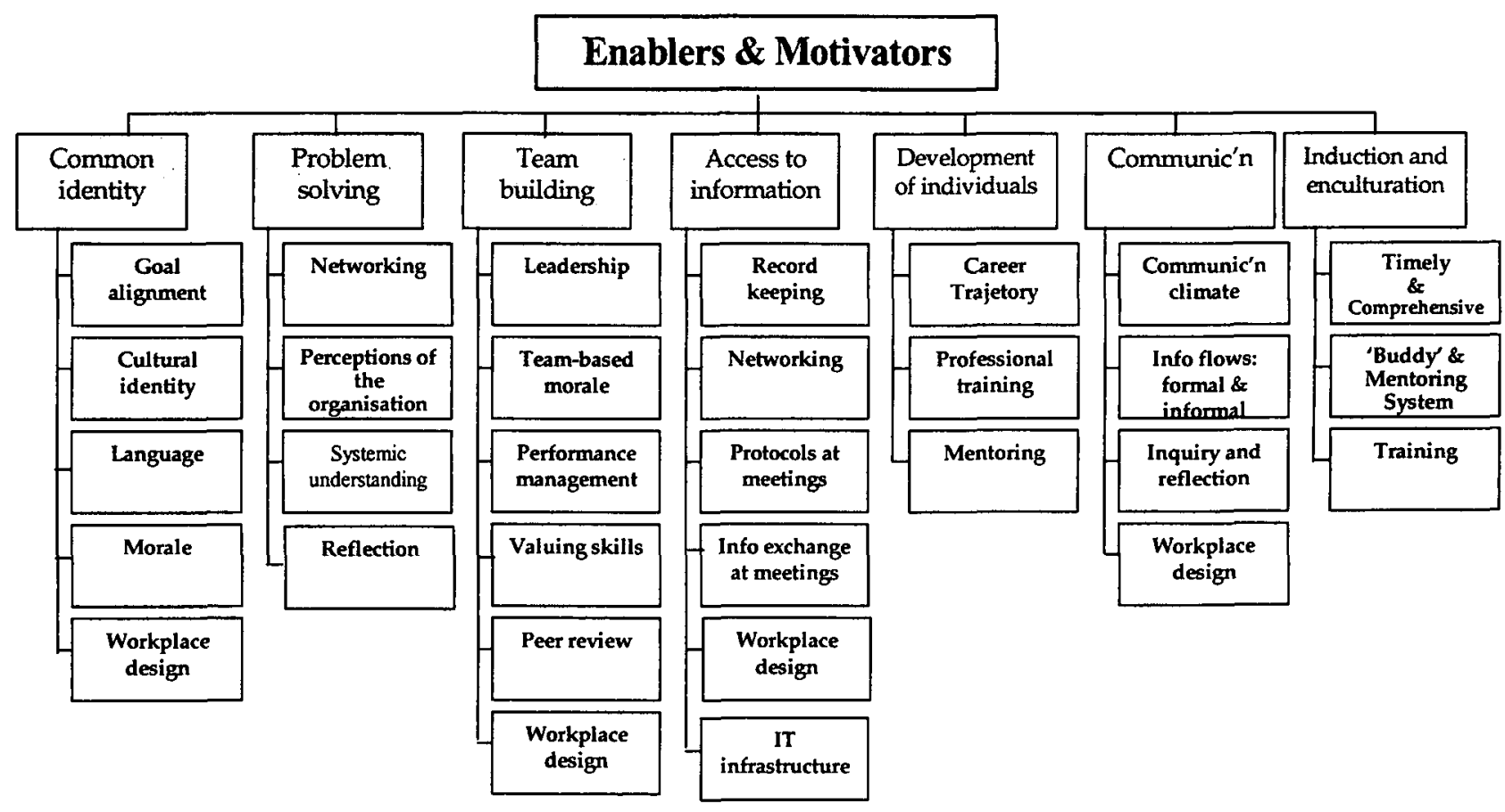

Figure 2 Social Learning Constructs

It is important to point out, that several of the motivators and enablers appear in more than one category as they play a role in supporting a number of social learning constructs (for example, 'Workplace Design' and 'Inquiry and Reflection'), and all of the identified factors appear to be inextricably intertwined (Ali, 2000; Warne, 2000).

\section{Common identity}

The ESLA findings identified a common identity as one of the important outcomes of having the following enablers and motivators in place. A common identity implies a similar way of describing and making sense of the world, of determining what is significant and important, and of how to use resources in the environment. Whilst a common identity and its sub categories are discussed as independent features in this paper, in reality they are not mutually exclusive since they significantly impact on one another.

\section{Goal Alignment Motivates and Enables}

Goal alignment is an important process within the work culture, particularly in terms of social learning as it impacts on work processes and on group cohesiveness. At the tactical environment studied, the team observed a strong and consistent degree of goal alignment within the community. In contrast, at the joint strategic headquarters facility, the research team found nothing uniform about the ways in which goal alignment takes place and that cohesiveness and work relationships varied in accordance to social positioning, hierarchy, and rank. Cohesive work arrangements were very much in evidence at the small team level, but less so as the teams expanded up the hierarchy of the organization. The ESLA team found that trust rather than goal alignment becomes more important as working environments become more risky and uncertain.

\section{Cultural Identity Motivates}

The term cultural identity refers to a staff member's sense of self in relation to the specific 'tribe' and 'tradition' to which they belong and how this distinctiveness applies in their workplace. Cultural identity is another important motivator for social learning. It impacts on the extent to which members of staff feel that they are part of the system or alienated from it. For the Australian military, cultural identity may mean Army, Air Force, or Navy cultures; while for civilians, their cultural identity may be as accountants, computing professionals, or public servants. At times, the ESLA team observed a clash of values occurring as cultural shifts took place. This threatened the extent to which 
staff felt that they were a part of the system and at times resulted in higher levels of alienation, and thereby reduced cultural identity.

\section{A Common Language Motivates and Enables}

Language is another important factor fundamental to the overall social leaming processes. By reflecting the social and political relationship between various members, language can impact on common identity. At the joint strategic headquarters re-organization workshop for example, it was stated that the Defence Executive had chosen the term 'staff' rather than 'division' to refer to sections within this workplace because the latter is seen as denoting separation and division between sections within the Branch. Language is also important in terms of creating a shared understanding among workers and their relationship to the wider organization. The importance of language was highlighted at the joint strategic headquarters induction, when one presenter said:

"Words are bullets. Never, never use imprecise language (when appraising performance). It can mean a career hit for somebody. ... There are subtle differences in language use between the services. Words that may signify praise in the Navy or Air Force may be poison in Army."

\section{Good Morale Motivates}

Morale has been a significant focus in the overall study because the research team found evidence of low morale being coupled with higher levels of alienation towards senior management. Such alienation has obvious implications for a common identity and thus for social learning. Perception of low morale has frequently been coupled with comments about not understanding the motivation or agenda of more senior staff. The team also found this lack of understanding not only affects morale, but also has an impact on trust, organizational cohesiveness, goal alignment and common identity, and consequently, on opportunities and motivation for social learning and the development and sharing of knowledge.

\section{Workplace Design Enables}

A particular problem within the joint strategic environment was that its staff was scattered amongst several sites within the city, a consequence of the reorganization that created it. The dislocation had impacted on many of the social learning constructs. There was strong evidence of reduced morale, problems in sharing information because some sites were unable to access file servers and classified networks, as well as the impact that the reorganization had on individuals' perceived power bases.

Many joint strategic personnel interviewed by the ESLA team stated that they enjoyed the spontaneity of face-to-face discussion on work related issues as they crop up, and so this became problematic for them when teams were not colocated. The converse also applied. Staff located in the smaller outposts, who often tended to be of junior ranks, often felt little identification with their organization. Several 'dis-located' staff members expressed significant confusion about their cultural and common identity, in some cases identifying more with the workplace with which they were based than their division within the joint headquarters workplace. This has serious implications for the effectiveness of social learning in these areas, and seriously inhibits knowledge sharing and knowledge transfer.

\section{Problem solving}

For knowledge workers in any organization, problem solving is a core activity. Importantly the process of problem solving fosters knowledge generation and thus social learning. For instance, routine tasks often need to be done slightly differently in different circumstances and in doing so involve an element of problem solving that requires generative learning (Lave \& Wenger 1991). Both the tactical and strategic settings studied provided numerous examples of this. The gap between formal or routine procedures and the ways in which people actually do their work was highlighted many times.

\section{Networking Enables Problem Solving}

An individual's network is one of their most important resources, as personal and social networks are an important means of acquiring, propagating and sharing knowledge. The individuals who comprise the network can make available their own knowledge, expertise and experience. In this way, the knowledge resources available to any one per- 
son, in their work and when problem solving, can be greatly increased. Staff at all settings studied relied upon their networks for problem solving.

Personal networks can also play a pivotal role in the propagation of knowledge. A few members of staff in the settings studied were seen to be systematic in passing on relevant knowledge to colleagues, and many others told the research team that, time permitting, they would pass information onto colleagues. As Davenport and Prusak(1998) claim, when those who are in a position of 'know-how' share their expertise they contribute to problem solving. Hence, personal networks were seen to function as channels supporting both 'information pull' and 'information push'. A good deal of effort can go into generating, maintaining and obtaining value from these networks. The research team was told that often, the primary benefit in attending a course or a conference was the new contacts that were made.

\section{Accurate Perceptions of the Organization Enable}

Individual and shared perceptions of the organization, and how they operate, provide an essential backdrop to problem solving within an organizational context. These perceptions may consist of deeply ingrained assumptions, generalisations, or even pictures or images that influence how people within an organization understand their organizational world and how they should act within it (Senge 1992).

The importance of these perceptions cannot be stressed enough, because they directly influence the construction of individuals' knowledge and understandings that they draw upon in their day-to-day-activities. One general example is appreciating the ways in which an organisation's formal rules and processes can be bent to achieve a desired outcome. This class of knowledge can empower people to solve problems by expanding the range of solutions that may be available, and by giving them confidence to improvise or innovate. Conversely, a lack of knowledge or incorrect perceptions will constrain the types of solutions that can be found and inhibit social learning.

\section{Systemic Understanding Enables}

Similarly, effective problem solving often requires a systemic understanding of organizational and interorganizational issues. Achieving a systemic understanding requires a holistic view of an organization and its interrelationships, an understanding of the fabric of relationships and the likely effect of interrelated actions (Senge 1992). Such systemic understandings are becoming more important for organizations as they position themselves to derive benefit from a constantly changing environment.

This systemic understanding of the workplace and its context was effectively applied to enhance work practices, in tandem with social learning, by a number of staff members observed in the research, but not by most. The military posting cycle and high staff turnover ensure a continuing need to make this class of information both readily available and to ensure that it is brought to the attention of all staff. Hence the need remains to develop, encourage and nurture systemic understandings of the relevant parts of the organization. The ESLA team was told on numerous occasions that an understanding of where one's job or a project fits into the big picture is an important element in job satisfaction and hence morale.

\section{Inquiry and Reflection Enables and Motivates}

Inquiry and reflection together are a powerful means of enhancing social learning and knowledge creation. Inquiries, or questions, can be triggered by problems or needs that require solutions or explanation. Reflection allows time for examination, contemplation and, often, resolution of the inquiries. To use a common metaphor, it is perhaps the best means for distinguishing between the forests and the trees of everyday working life. Inquiry and reflection involving more than one staff member is an opportunity for both creating and sharing knowledge.

Some of the processes at the tactical setting studied, such as the mission debrief held after each mission, were seen to include elements of inquiry and reflection. In contrast and with very few exceptions, most of the staff interviewed at the strategic level headquarters regretted that their high workloads meant they had insufficient time for inquiry and reflection at the workplace, either individually or as a group. One pertinent comments was:

"We spend so much time in the detail on the process that we're in, that we rarely give ourselves the opportunity to think strategically about what we're doing and to really determine the priorities of where our effort is worthwhile and where it isn't. I think you need to do that both individually and at a group level again with the people that you work with." 
In order to effectively solve problems and innovate, time for inquiry and reflection must be factored into the workplace. Staff are generally more motivated to do so if they know that inquiry and reflection is recognised as a valid and valuable use of their time.

\section{Team building}

Very few people work by themselves and achieve results by themselves. So the people who interact together and yet have different tasks and responsibilities need to understand what each of them are trying to do, why they are doing it, how they are doing it, and what results to expect. In this relationship of interdependencies, communication and trust play vital roles (Drucker, 1999).

\section{Good Leadership Enables and Motivates}

In general, the leaders and managers of the areas studied were innovative and they motivated and developed their staff. The research team witnessed many meetings where leaders used inclusive language, were receptive to new ideas and openly shared their thinking processes and imperatives. They empowered others and the team's mutual respect was evident regardless of rank.

"I've tried to engage people, involve them in the decision making process, keep people informed because I think people who are informed will be better participants and contribute better to whatever it is that we are trying to achieve, and where appropriate collegiate. Sometimes however, a more directive style is necessary."

The ESLA team spoke to many staff members who expressed their appreciation for the accessibility of their leaders, their non-judgemental approach and their courage in admitting their shortcomings. Such qualities and atmosphere build up trust, and encourage collaboration and teamwork. The military leadership also exhibited two other motivating dimensions: a willingness to demonstrate that staff are highly valued and a willingness to acknowledge expertise and knowledge regardless of rank. This was particularly evident in the tactical setting.

\section{Strong Team-based Morale Motivates}

'Team spirit' and 'team cohesiveness' are both important values within the work culture and work ethic in the settings studied. In some of the settings studied, the attitude of 'them and us', as well as a feeling of being undervalued, was clearly prevalent. These individuals did not identify themselves as team members of the unit they belonged to, and some stated they were not encouraged to operate in a coordinated way to support organizational goals. This was because they did not see the significance of their particular tasks to the overall goals of the organization and this feeling was exacerbated by the geographic distribution of team members. This was a serious de-motivator, and these staff members were not keen to learn or share their knowledge with other team members.

However, some examples of effective teamwork and team spirit were evident in the settings studied. The researchers observed instances where teamwork was well integrated into daily work and where people worked collaboratively. Such teams were goal oriented and bound by achieving good results. There were not only teams in structure, but in spirit. They were usually formed within individual sections and were led and energised by a leader who saw his or her role as fostering team cohesiveness. Members of these teams were co-located and made information (such as calendars, contacts, and computer work folders) available to one another. This not only contributed to knowledge sharing among team members and aided communication, but it also emphasised trust within the team. In these areas, morale was perceived to be high. People worked together happily and looked forward to socialising together. Regular social functions were organised for the workplace teams.

\section{Constructive Performance Management Enables}

Assessment, reporting and performance management form a significant part of the overall management of military personnel throughout their careers; however, if not handled constructively it may have adverse impacts on team spirit and thus on social learning. The outcome of a performance report often determines the prospects of one's career progression. The ESLA team was told that a poor performance rating, at a critical point in one's career, would severely reduce the prospects for promotion. This strong emphasis on individual performance management may be divisive as it may influence a proportion of individuals to focus on achieving their individual goals at the expense of assisting their team to achieve its goals. Team-based performance management systems are being discussed, but have yet to gain wide acceptance. 
For the military, the performance cycle is annual. It was reported to ESLA team members that there was often a lack of clear communication about performance expectations. Some of the people interviewed were unclear about what was expected of them and what was stated in their duty statements. An annual performance appraisal appears to be too long to wait for recognition of good work and too late to correct a performance problem. Clear communication about performance expectations can contribute not only to achieving organizational goals but also to appropriately directed social learning and knowledge development (Morgan, 1989; Wood, 1989).

\section{Valuing Skills Motivates and Enables}

At the tactical setting studied, the skills of individuals were seen to be highly valued. Current expertise and competence were often seen to take precedence over rank. This type of interaction is acknowledged as an important cultural requirement for organizations that wish to further their generative learning (Bokeno, 2000; Isaacs, 1999). The researchers were told that the attitude had changed over previous years. Before that change, senior officers believed that they must maintain the pretence that they were superior in all spheres of performance, to maintain their authority. However, informants explained that the results of that attitude were counterproductive. A senior officer stated that:

"There has been a huge change with the culture regarding senior and junior officers. Previously, junior officers would not go out of their way to help them - the attitude was, if you knew it all, then do it yourself. It couldn't be more different now. They will offer help when they see it is needed. You simply need to say what you require. They respect the fact that we have other jobs to do and are not as current as we once were."

One way of valuing skills and increasing morale is to publicly acknowledge outstanding work. Key informants stated that public recognition of good work was scarce in the strategic settings studied. Many personnel told the research team that a written or verbal word of praise, a pat on the back often means more, for example, than a pay raise "praise is better than money".

\section{Peer Review Enables}

For the military, peer review is an important way of sharing expertise and problem solving. When done constructively, it also has a positive effect on team building because it demonstrates that colleagues are willing to contribute to achieving an individual's tasks and goals, and peer review helps to build and sustain relationships and to generate trust.

Peer review was seen to form an essential part of team building at the tactical headquarters, and to a lesser extent, at the strategic settings. At the tactical setting, the mission debrief is a major component of peer review. Each mission is analysed to see how well its objectives were met, and how each individual's actions contributed towards the success or failure of that component of the mission. Furthermore, this is done without singling out individual's failures or assigning blame. The importance of peer review was stressed by a senior officer who said "Interaction with peers is an important way of getting feedback, constructive criticism and validation". Certainly, constructive peer review enables knowledge sharing and social learning.

\section{Effective Workplace Design Enables}

Workplace design was seen to have many negative impacts on social learning. Staff located at small isolated outposts often felt excluded by their colleagues. The ESLA team was told about a number of instances when these workers were overlooked or not informed about meetings or other events. Several staff members expressed confusion as to who was in their line management, or even their place in the ADO. Those teams where staff was co-located demonstrated the highest team-based morale and team spirit. However, whilst collocation can be helpful, it is insufficient on its own to guarantee successful team building.

\section{Access to information}

The easy availability of corporate information has a direct input into knowledge acquisition and generation, and thereby, social learning. Information, therefore, is an important organizational resource which, if properly managed, can lead to improved decision making and increased productivity. 


\section{Good Records Keeping Enables}

The research team observed that general familiarity with corporate records keeping procedures in the settings studied was quite poor and adherence to formal process was almost non-existent. Records management and access to information contained in paper records posed a problem. Some people had developed their own personal records keeping systems but there was little uniformity in these, and no adherence to file naming conventions and standards. Consequently, there seemed to be little faith in the integrity of organizational records. Those areas where there was good local practice in records management were seen to be very much the exception. As one informant stated:

"I believe that physical files in the ... are no longer managed well because their management has been farmed out to outside bodies. With the file clerks there was consistency of procedures but the file clerks are no longer part of the procedure."

Furthermore, the preference for accessing and transferring information electronically seems to be growing, and the use of electronic tools for communication and decision making is prevalent at all levels. The issue of electronic records, particularly e-mail messages containing evidence of business transactions, posed problems not only in the setting studied but also in the ADO at large. Research reported in the professional literature on records management (Enneking, 1998; Henricks, 1999) suggests that the capture of email messages into a records management system offers the best solution to this problem. However, in the absence of organization-wide protocols, technology is only as good as the people using it:

"I think we have problems with passing on information in the organization as a whole... You know we go upstairs and ask people where they save their work to and you ask five people you'd find different answers. We have an information management problem right from the outset. Where do you save your e-mails? Well I save them somewhere. Where do you save yours? I put mine in a public folder. Where do you save yours? I put them into private folders. It's corporate information.... we have an information management problem and therefore passing on information becomes quite difficult because everybody does it in a different way."

\section{Personal Networking Enables}

Personal networks from previous postings, as well as newly acquired contacts, play a pivotal role in knowledge propagation. New knowledge often begins with the individual and making personal knowledge available to others is the central activity of knowledge creating organizations. Through conversations people discover what they know, what others know and in the process of sharing, new knowledge is created. Technologies such as e-mails, faxes, and telephones are invaluable aids in the process of knowledge sharing, but they are only supporting tools. Knowledge sharing depends on the quality of conversations, formal or informal, that people have. Webber (1993) describes this process: "Conversations - not rank, title, or the trappings of power - determine who is literally and figuratively "in the loop' and who is not."

\section{Protocols at Meetings Enable}

Meetings are another means of accessing information and those that were observed in the different settings studied varied significantly in format and the protocols in place. The meetings in the tactical environment were seen to be more conducive to social learning than those at the strategic environment. At the tactical headquarters, mission related meetings were observed to be excellent opportunities for learning. Strict protocols were observed at such briefings allowing participants to discuss errors or problems encountered during missions without assigning blame or shame to individuals. As well as providing access to information, this facilitated the sharing of mistakes and the sharing of responsibility for solutions. Even briefings where the main aim was information transfer from senior to junior staff ensured that staff were invited to speak to topics, discuss tactics or to identity potential objectives. The ESLA team had been told that, prior to the introduction of these protocols, the debriefs commonly became very heated and it was not uncommon for fighting to take place. A similar meeting protocol, although not as consistently adhered to, was observed in the single service strategic headquarters. However, there were few equivalent meetings at the joint strategic headquarters, other than some induction meetings and briefings. In an environment which, in many ways seemed the most complex and ambiguous, learning how to do the job was not given quite the same priority. 


\section{Effective Information Exchange at Meetings Enables}

Many meetings at the settings studied were held with the stated aim of propagating and exchanging information. Information transfer is generally considered to be an uni-directional process, while information exchange is a two-way or multi-directional process.

While meetings observed in the joint strategic environment tended to have a basic structure, their purpose appeared to be more for information transfer than for information exchange or information sharing. Conversely, in both the single service settings, meetings usually presented ample opportunities for information exchange, brainstorming and group reflection. However, the ESLA team observed a vast difference among different sub-units in the way information was disseminated at meetings, so this protocol was very much dependent on local practice, which was generally determined by the manager. Some managers did not have regular meetings at all. They conveyed information passed on from their superiors to their subordinates on an ad hoc basis, expecting them, in turn, to disseminate information to their respective subordinates. The research team was told that this 'multi-step' means of disseminating information compromised the content (inevitably filtering of information took place) as well as accuracy of information. One survey respondent stated that:

"Whilst at a social level members of ... interact well, there is very poor group cohesiveness in the work environment. I believe that this is in part due to the failure to conduct regular progress / section meetings at lower levels and the failure to clearly identify who is doing what."

Whilst it is sometimes necessary to have meetings for information transfer only, knowledge transfer and social learning is more effective when there are opportunities for an open information exchange and dialogue at meetings and where questions can be asked, views expressed and problems raised without fear of blame or ridicule.

\section{Workplace Design Enables}

Workplace design is also an enabler for the category of Access to Information because it impacts on the ways by which individuals can access information. In the first instance, it is important that individuals know that some types of information exist. It is more likely to be used if it is readily accessible rather than if it is hidden or scattered across many offices. This problem is exacerbated by geographical distribution of units within the organization. This leads to a greater reliance on personal networks and on obtaining information from people rather than from documents and centralised sources. In the worst cases, it can lead to a loss of interest in information seeking or information verification.

\section{Good IT Infrastructure and Protocols Enable}

The ESLA team observed that information access due to failings in the IT infrastructure inhibited access to information within the strategic settings. Hardware crashes and system failures were common. It was especially a problem for those individuals who were not co-located in the central headquarters. They lacked access to classified networks and to the main information resources stored on shared hardware drives; similarly, those staff located within the central headquarters had difficulty accessing some shared information resources of outposted staff.

Another issue that was often brought to the team's attention was the difficulty in finding information on the shared drive. Since there was no specific person responsible for maintaining information on the shared drive and for naming folders, it was left to the discretion of the document originator where each document would be stored. The research team observed that in some units, people were more precise in giving explicit names to documents and folders but this practice was not uniform across the whole of the headquarters.

\section{Development of individual expertise}

The acquisition and development of individual expertise is an integral part of knowledge development and social learning and there are a number of enablers that have been observed to facilitate this. 
AJS Special Edition
Knowledge Management
December 2001

\section{Career Trajectories Enable}

An individual's career trajectory describes the positions, roles and experience that he or shehas accumulated, up to and including the position currently held. Whilst not excluding personal experiences outside of a work or training context, a well-designed career trajectory generally equips an individual with the skills, experience, maturity and personal networks needed to successfully fill a particular position or role. Appropriate career trajectories facilitate social learning because they provide a foundation of knowledge upon which an individual can become fully productive more quickly, and as a consequence they are more able to construct new knowlcdge. Good career management and thoughtful recruitment and selection procedures could serve the same purpose in civilian organizations.

\section{Professional Training Enables and Motivates}

Appropriate and timely professional training is a significant component of the development of individual expertise and, therefore, a fundamental enabler for acquiring and generating new knowledge. Generally, the military excel at supporting their officers through in-house, graduate and postgraduate training. Training courses are also important to furthering individuals' expertise, as well as for forming the personal networks that subsequently develop. However, in times of budgetary constraints, training money is often the first to go, with damaging consequences for the organization's ability to learn and develop their knowledge.

The ESLA team observed that numerous individuals at the strategic settings studied believed there was a lack of funds available for training. This perceived shortage of funding could have a direct impact on the trust that staff have for management. Over many years, researchers (for example: McCauley \& Kuhnert, 1992 and Argyris, 1973) have found that a general trust in management is associated with professional development opportunities at work. The implication is that an employee's sense of trust is fostered when the supervisor provides career growth opportunities because it authenticates the supervisor's commitment to that employee's professional development. This is important to social learning because trust plays a key role in collaboration and teamwork. As it turned out, in one of the strategic settings, there were ample funds available for training, but the availability of these funds was not effectively communicated to those who would have taken advantage of them.

\section{Mentoring Enables and Motivates}

A degree of mentoring may be an important element in the development of individual expertise. Mentoring is regarded as an effective method of assisting staff development, especially for junior staff. At the tactical headquarters, for example, a degree of mentoring was seen to be built into the training program.

In terms of developing a career trajectory, the knowledge acquired through mentoring may also be important when individuals want to prepare themselves for specific roles in the future. The active management of junior officers' careers in the tactical environment provided another avenue for mentoring. The ESLA team, however, saw little evidence of systematic attempts at mentoring within the strategic environments although a mentoring program was on the 'wish list' of many staff members:

“...it might range from ex-politicians, ex-Secretaries, senior members of the Strategy Committees, or something like that - mentors that have previously been through the system, just saying, 'Hey, look, this is these are the hidden rules about how you do the thing".

\section{Communication}

Communication is one of the social learning constructs essential to effective learning and knowledge management within an organization, and to the effective functioning of any workplace.

\section{A Supportive Communication Climate Enables and Motivates}

Supportive communication climates are characterised by an open and free exchange of information, constructive conflict management procedures, and a high degree of worker involvement in solving organizational problems (Larsen \& Folgero 1993). A supportive communication climate includes a culture of sharing knowledge, treating each other with respect, and breaking down cultural barriers arising out of inaccurate stereotyping. Research has established the link between supportive organizational communication climates and generative learning as well as with higher levels of organizational commitment (Bokeno 2000; Ruppel 2000; Guzley 1992). . 
In both the single service environments studied, a supportive communication climate predominated. In the joint environment, however, the research team more often observed examples of defensive communication behaviour. Findings from semi-structured interviews with these staff also indicated lower levels of organizational commitment. In such settings, social learning and knowledge sharing is inhibited.

\section{Effective Formal and Informal Information Flows Enable}

Within a tactical environment such as the single service tactical headquarters studied, the communication flows, both formal and informal, are the lifeblood of the organization. It simply could not function without the meetings, briefings, email, telephone calls and conversations that take place. These activities provide information, align goals between individuals and different parts of the organization and help to coordinate their activities. When communication channels and information flows break down, as was observed several times at a high-stress military exercise, the consequences may be severe. The situation is similar in the strategic environments even though many of the time pressures are not as dire.

The data gathered suggests that formal information flows were not always effective within the strategic organizations. Information flowing from the top levels of the organization to the lower levels was perceived to be excessively filtered and fragmented in the process. But information flows from the lower level to the senior ranks, and lateral information flows, were seen to be particularly ineffective.

Informal information flows, however, seem effective in all the settings studied. On numerous occasions, it was pointed out that informal meetings, such as, morning teas or lunches provided an invaluable forum for exchange and transfer of information. In fact, these informal gatherings were a preferred means for communication and exchange of views by many staff. Apart from socialising, the team was told, a shared lunch provided a forum where people could talk informally about work and decisions were often made on the basis of information acquired at these lunches. People were regretful, however, that under current workloads, a lunch hour is often used to catch up on work and thus there is less and less networking between colleagues.

\section{Inquiry and Reflection Motivates and Enables}

An important element of generative learning is for organizational members to be able to engage in dialogue, which is open and is based on inquiry and reflection. A supportive communication climate is a prerequisite for such dialogue and it requires learning how to recognise defensive patterns of interaction in teams that undermine learning (Senge 1992). However, an additional and obvious requirement for such dialogue is having the time to engage in it. As stated earlier, on numerous occasions, the ESLA team encountered comments from the strategic headquarters staff that there is little time to reflect, leam from experiences, whether they be successes or failures, and generally discuss work matters. The comments were often made with an indication of bitterness and workload was attributed as a factor preventing people from setting some time aside for thinking and reflection.

Many of the processes at the tactical headquarters, from mission debriefs to tactics meetings and training, demonstrated that they recognised the importance of inquiry and reflection at a group level. As well as improving the quality of the reflection, group reflection also contributes to the development of a common identity and a shared understanding amongst the participants.

\section{Workplace Design Enables}

The issue of workplace design and its impact on team and network building, and on accessing information necessary to getting one's job done, arose repeatedly during the study. Numerous interviewees were aware that physical location and proximity to each other had the potential to promote the transfer of pertinent knowledge. Indeed, the point was even made that in addition to more quickly obtaining answers to questions about particular tasks, an open plan workplace enabled one to tap into pertinent information by overhearing others' conversations. Officers at the tactical headquarters settings believe that opportunities for learning have significantly decreased, citing the decrease of open plan areas as a factor:

"These days this has all but gone out of the window. The crew rooms are no longer used in the same way.

Nowadays, they all have desks and work separately. This change has meant that the learning, which used to take place informally in the crew room, happens less frequently."

However, as Davenport and Prusak (1998) point out, co-location in itself does not guarantee the sharing of knowledge; a common training or experience, or at least a common language, is essential. Unless individuals are prepared 
to ask and answer questions of one another, or to even just chat with each other, the knowledge advantage provided by open plan workplaces will be lost. An example of this was brought to the research team's attention when told that two workers had been co-located for three months before they realised that they were both working on the same project.

Conversely, many staff members of the strategic headquarters setting considered the open plan arrangement noisy and an inhibitor of effective communication, as the following comment illustrates:

"If the environment was such that a group of people who worked together were located together and had some form of privacy to do their work as a group, that would be fine but when you're all lumped together and everybody can hear what everybody is saying and everybody's saying a hundred things at the same time, sometimes it can be an absolute nightmare."

\section{Induction and Enculturation}

Orientation of new employees is often considered to be one of the most overlooked aspects of employee training (Ganzel, 1998; Tyler, 1998). Like appropriate career trajectories and professional currency, effective induction and enculturation programs facilitate social learning by providing a foundation of knowledge upon which the individual can become fully productive more quickly and as a consequence they are more likely to assimilate new knowledge. Good induction, however, is more that just an introduction to new job and workmates; it is a way of helping people find their feet and shape their attitudes and expectations (Dunford 1992; George \& Cole, 1992). There are numerous advantages that come from good induction programs. These include morale building, minimisation of misunderstanding (because rules and regulations have been clearly explained), establishment of good working relationships, reduction of anxiety, and reduction of inefficiency.

\section{Comprehensive, Timely, Induction Programs Enable}

Induction, or the perceived lack of it, was a problem in the joint strategic setting studied. Although not everybody interviewed was explicitly critical about the lack of job induction, some felt frustrated because ofien they had to labour to find obvious organizational information required for their work. This "discovery learning", as it was referred to, was regarded as very time consuming and seemed to lead to poor morale, frustration, and it negatively influenced people's perceptions about the organization. The plight of some informants was made clear in the following remarks: "...there was just no guidance, no handover, nothing about where this paper was at and what I should do with it."

"We had no ... handover in terms of the status of projects that we were going to assume responsibility for. No handover in relation to file or information management within the section. So we just foraged. We're still foraging."

Timing is one of the most important elements of employee job induction. If the employees have to wait for weeks to be introduced to the job and the organization, they have been largely unproductive for that period of time. An issue that emerged from the interviews with staff in the strategic headquarters was the relationship between meaningful and timely induction and subsequent job satisfaction. What was also interesting was that those who were not properly inducted or enculturated into the organization saw no need and responsibility to actually prepare any form of handover for anyone who may take over their position in the future. If learning is to occur, a timely and comprehensive induction program would provide a solid ground to begin this process. 


\section{'Buddy' and Mentoring Systems Enable and Motivate}

Although highly desirable, when a new posting cycle begins, it is not always feasible to conduct an induction program that all new starters can attend. This is even harder to do in a civilian organization where new staff can start at any time during the year. In the interim, a 'buddy' or 'mentoring' system could fill in the gap. A 'buddy', would be an assigned, experienced workmate who could be available to answer questions and assist the orientation of new members during the initial few weeks. Some interviewees mentioned that a colleague acted informally as a 'buddy' when they first came to the headquarters, and that they found this to be invaluable to settling into a new job and to effective learning. For instance, one person commented:

"Well, because [name] did the job before, and was pretty much my bible for the first three months so I was relying on [name] for a lot of stuff".

Similarly, mentoring is regarded as an effective method of staff enculturation and development. Its advantages include helping to align staff with organizational goals, and providing a context for the transfer of knowledge between senior and junior staff (Davenport \& Prusa Prusak 1998).

\section{Early Training Enables}

The ESLA team was repeatedly told that early training is an important part of effective induction and enculturation. It is an opportunity to learn the explicit knowledge that is taught as part of formal training. It is also an opportunity to be exposed to the attitude and cultural perceptions of colleagues and peers.

\section{ORGANISATIONAL VALUES}

The organisational values that arise from the identified motivators and enablers, in turn facilitate the effective application of the same motivators and enablers. This recursive relationship is characteristic of the architecture that is emerging from the findings of this research. While motivators form a sound foundation and enablers provide the bricks or building material, the values are the mortar that binds them all together. Furthermore, each of the factors is intertwined with, and dependent on, the others for the organization's social learning success and continued robustness (Ali, 2000; Warne, 2000).

The overarching organisational values that emerged from the research nurture, and are nurtured by, the motivators and enablers identified. These values include:

- empowerment (where empowerment of staff also makes them accountable);

- trust (which entails mutual respect);

- forgiveness (in terms of allowing personnel to take reasonable risks, forgiving mistakes and facilitating knowledge construction on the basis of lessons learnt)

- cultural cohesiveness (in terms of common identity, shared goals and a shared understanding)

- commitment (which includes a mutual commitment and loyalty from the employee to the organisation as well as the organisation to the employee)

- openness of the decision making process and

- a culture of information sharing.

\section{CONCLUSION}

The implication of this study is that organisations seeking to improve information sharing and knowledge generation need to develop a greater awareness of the processes and strategies of organisational learning. Organisational knowledge is distributed across functional groups and its generation and continual existence is dependent on the overall communication climate, which is embedded in the organisational culture.

Change and information overload are part of the normal operating conditions of most organizations today, and are likely to remain so for the foreseeable future. Organisations need to be able to renew themselves constantly and respond creatively to the market forces of the information economy while maintaining their knowledge assets. An organizational culture recognising the value of knowledge and its exchange is a crucial element in whether knowledge work is successfully carried out or not. Such a culture provides the opportunity for personal contact so that tacit knowledge, which cannot effectively be captured in procedures or represented in documents and databases, can be transferred. In a culture that values knowledge, managers recognise not just that knowledge generation is important for organizational success but also that it can be nurtured with time, and space (Davenport \& PrusaPrusak, 1998). 
While the research described in this paper is continuing, and new findings may emerge, it is possible to reach some conclusions about effective social learning in the ADO, as identified to date. The ESLA research has identified a substantial number of complex and interwoven social learning constructs, and the motivators, enablers and values that support social leaming and facilitate effective knowledge management holistically. The results of these studies strongly suggest that the interplay between human, social and organizational issues within an organization must be considered first, to effectively facilitate social learning and knowledge management. The study findings to date indicate that information sharing and subsequent knowledge generation is successful where interactive environments are cultivated before technological solutions are considered. Technology alone, however well designed it may be, is unlikely to produce effective knowledge management solutions in the absence of appropriate attention to such issues. Although some of these issues are specific to a military organization, most are equally applicable to any organization that is attempting to improve its learning capability and knowledge management.

\section{ACKNOWLEDGEMENTS}

The authors wish to acknowledge the vital contribution made to this work by Dr Jennie Clothier whose vision made it possible and to all the Defence personnel who participated in this study. Thanks are also due to the two reviewers of this paper who gave helpful and constructive advice.

\section{COPYRIGHT}

Commonwealth of Australia (c) 2001. Leoni Warne, Irena Ali, Celina Pascoe and Katerina Agostino for, and on behalf of, the Commonwealth of Australia assign a non-exclusive licence to use this document for personal use and in courses of instruction provided that the article is used in full and this copyright statement is reproduced.

\section{REFERENCES}

Ali, I., Pascoe, C., Warne, L., Gori, R., and Agostino, K. (2000). Enabling learning: A progress report on the Enterprise Social Learning Architecture Task, Proceedings of the 5th International Command and Control Research and Technology Symposium, Canberra, Australia.

Argyris, C. (1973). On organisations of the future. Beverley Hills, CA: Sage.

Bokeno, R. M. and V. W. Gantt (2000). "Dialogic Mentoring." Management Communication Quarterly, November, Vol 14, no 2, pp 237-270.

Davenport, T. H. and L. Prusak (1998). Working knowledge: how organisations manage what they know. Boston, Massachusetts, Harvard Business School Press.

Drucker, P. (1999). "Managing oneself." Harvard Business Review, March-April, pp 65-74.

Dunford, R. W. (1992). Organisational behaviour: an organisational analysis perspective. Sydney, Addison Wesley.

Enneking, N. E. (1998). “Managing email: working toward an effective solution.” Records Management Quarterly, Vol 32, no 3, pp 24-43.

Ganzel, R. (1998). "Elements of a great orientation."Training, Vol 35, no 3, pp 56.

George, C. S. and K. Cole (1992). Supervision in action: the art of managing. Sydney: Prentice Hall.

Guzley, R. M. (1992). "Organizational climate and communication climate: predictors of commitment to the organization." Management Communication Quarterly, Vol 5, no 4, pp 379-402.

Harvey, L. and M. D. Myers (1995). "Scholarship and practice: the contribution of ethnographic research methods to bridging the gap." Information Technology \& People, Vol 8, no 3, pp 13-27.

Henricks, M. (1999). "Sorting out electronic filing." Office Systems, Vol 16, no 2, pp 30-34.

Isaacs, W. (1999). Dialogue and the art of thinking together. New York: Currency Doubleday.

Jordan, B. (1993). Ethnographic Workplace Studies and Computer Supported Cooperative Work. Interdisciplinary Workshop on Informatics and Psychology, Scharding, Austria.

Larsen, S. and I. S. Folgero (1993). "Supportive and defensive communication." International Journal of Contemporary Hospital Management, Vol 5, no 3, pp 22-25.

Lave, J. and E. Wenger (1991). Situated learning: legitimate peripheral participation. Cambridge,UK: Cambridge University Press. 
Linger, H. and Warne, L. (2001). "Making the invisible visible: modelling social learning in a knowledge management context", Australian Journal of Information Systems, Special Edition on Knowledge Management.

McCauley, D. P. and K. W. Kuhnert (1992). The professional manager. New York:McGraw-Hill.

Miles, M. B. and A. M. Huberman (1994). Qualitative data analysis: an expanded sourcebook. London: Sage.

Morgan, T. (1989). Performance management -- the missing link in strategic management and planning. Public Sector policies for the 1990's. D. C. Corbett. Melbourne, Public Sector Management Institute, Faculty of Economics and Politics, Monash University, pp 243 - 250.

Ruppel, C. P. and S. J. Harrington (2000). "The relationship of communication, ethical work climate, and trust to commitment and innovation." Journal of Business Ethics, Vol 25, pp 313-328.

Senge, P. M. (1992). The fifth discipline: the art and practice of the learning organization. Sydney: Random House.

Tyler, K. (1998). "Take new employee orientation off the back burner." HR Magazine, Vol 43, no 6, pp 49-57.

Warne, L. (2000. "Understanding organisational learning in military headquarters: findings from a pilot study"", Australian Journal of Information Systems, Vol 7, no 2, pp 78-87.

Webber, A. M. (1993). “What's so new about the new economy?" Harvard Business Review, Jan-Feb, pp 24-42. 